\title{
CICLO BIOLÓGICO, EXIGÊNCIAS TÉRMICAS E PARASITISMO DE Muscidifurax uniraptor EM PUPAS DE MOSCA DOMÉSTICA
}

\author{
Marcílio José Thomazini*; Evôneo Berti Filho² \\ ${ }^{1}$ Embrapa Acre - C. P. 392 - CEP:69901-180, Rio Branco, AC. \\ ${ }^{2}$ Depto. de Entomologia, Fitopatologia e Zoologia Agrícola, USP/ESALQ, C.P. 9, 13418-900, Piracicaba, SP. \\ *Autor correspondente: <marcilio@cpafac.embrapa.br>
}

\begin{abstract}
RESUMO: Os parasitóides pupais estão entre os principais responsáveis pela redução populacional de mosca doméstica em aviários, e Muscidifurax uniraptor Kogan \& Legner (Hymenoptera: Pteromalidae) é um dos principais parasitóides presentes nestes ambientes no Estado de São Paulo. O objetivo deste trabalho foi determinar a duração do ciclo biológico, as exigências térmicas e o parasitismo de $M$. uniraptor em pupas de Musca domestica L. (Diptera: Muscidae) em temperaturas constantes. Os testes foram realizados em câmaras climatizadas nas temperaturas de $18,20,22,25,28,30$ e $32 \pm 1{ }^{\circ} \mathrm{C}, 70 \pm 10 \%$ U.R. e $14 \mathrm{~h}$ de fotofase. Em cada câmara 200 pupas de mosca doméstica, com 24 a 48 h de idade, foram expostas a 40 parasitóides fêmeas partenogenéticas, com 0 a $24 \mathrm{~h}$ de idade, por $24 \mathrm{~h}$. A temperatura influenciou o desenvolvimento e a reprodução de $M$. uniraptor. O menor valor do período de ovo a adulto foi a $30^{\circ} \mathrm{C}$ (17 dias) e as porcentagens de parasitismo e de emergência de descendentes foram maiores a $28^{\circ} \mathrm{C}$, com 87 e $63,5 \%$, respectivamente. A temperatura base encontrada para fêmeas do parasitóide foi de $9,43^{\circ} \mathrm{C}$, com uma constante térmica de 366,62 graus-dia (GD). A elevação da temperatura diminui a duração do período de ovo a adulto (entre 18 e $30^{\circ} \mathrm{C}$ ) e aumenta o parasitismo (entre 18 e $28^{\circ} \mathrm{C}$ ) de $M$. uniraptor. A faixa de temperatura entre 28 e $30^{\circ} \mathrm{C}$ é considerada a mais adequada para criação de $M$. uniraptor em pupas de mosca doméstica.
\end{abstract}

Palavras-chave: M. domestica, Insecta, controle biológico, parasitóide pupal, temperatura

\section{LIFE CYCLE, THERMAL REQUIREMENTS AND PARASITISM OF Muscidifurax uniraptor ON HOUSE FLY PUPAE}

\begin{abstract}
The pupal parasitoids are among the most important organisms responsible for house fly population reduction in poultry farms. Muscidifurax uniraptor Kogan \& Legner (Hymenoptera: Pteromalidae) is one of the main parasitoids that occur in poultry farms at São Paulo State, Brazil. The objective of this study was to determine the life cycle duration, the thermal requirements and the parasitism of $M$. uniraptor on Musca domestica L. (Diptera: Muscidae) pupae under constant temperatures. The experiment was realized in climatized chambers at seven temperatures $\left(18,20,22,25,28,30\right.$ and $\left.32 \pm 1^{\circ} \mathrm{C}\right), 70 \pm 10 \% \mathrm{R} . \mathrm{H}$. and $14 \mathrm{~h}$ of photophase. In each chamber 200 house fly pupae ( 24 to $48 \mathrm{~h}$ old) were exposed to 40 partenogenetic female parasitoids ( 0 to $24 \mathrm{~h}$ old) for $24 \mathrm{~h}$. The temperature influenced the development and reproduction of $M$. uniraptor. The lowest value of the egg-adult period was 17 days, and occurred for $30^{\circ} \mathrm{C}$ and the parasitism and progeny emergence were highest at $28^{\circ} \mathrm{C}(87$ and $63.5 \%$, respectively). The lowest temperature threshold for the parasitoid females was $9.43^{\circ} \mathrm{C}$, with a thermal constant of 366.62 degree-days. The temperature elevation reduces the duration of the egg to adult period (between 18 and $30^{\circ} \mathrm{C}$ ) and increases the parasitism (between 18 and $28^{\circ} \mathrm{C}$ ) of $M$. uniraptor. Temperatures between 28 and $30^{\circ} \mathrm{C}$ are the most adequated for rearing $M$. uniraptor on house fly pupae.

Key words: M. domestica, Insecta, biological control, pupal parasitoid, temperature
\end{abstract}

\section{INTRODUÇÃO}

O manejo dos excrementos e o uso adequado de inseticidas seletivos são as táticas principais para preservar e aumentar a população de inimigos naturais em aviários. No entanto, o aumento desses organismos através de liberações periódicas, principalmente parasitóides, também tem se mostrado uma alternativa viável (Axtell, 1986). Para se assegurar que essas sejam eficientes, vários fatores devem ser considerados, como, por exemplo, a resposta desses insetos benéficos à temperatura, fator ambiental muito importante na determinação da distribuição dos organismos e que afeta diretamente a sua biologia. Trabalhos demonstrando a influência da temperatura no desenvolvimento de parasitóides pupais de mosca doméstica, que são os principais responsáveis pela redução populacional de dípteros nocivos, na fase de pupa, em aviários, foram feitos por Axtell \& Arends (1990).

A duração do período de ovo a adulto de Spalangia endius Walker e Muscidifurax raptor Girault \& Sanders foi estudada por Ables et al. (1976), verificando que o primeiro parasitóide não completou seu ciclo quando os imaturos foram submetidos as temperaturas de 12,8 e $15,6^{\circ} \mathrm{C}$, já o segundo não completou seu ciclo a 12,8 e a $35^{\circ} \mathrm{C}$. Diferenças no comportamento de oviposição dessas duas espécies também ocorreram, sendo que os valores mais baixos de temperatura foram mais favoráveis a $M$. raptor (Ables \& Shepard, 1976). Costa (1995) quantificou os efeitos da temperatura na 
biologia do parasitóide Spalangia gemina Boucek, relatando que a duração do desenvolvimento de ovo a adulto foi inversamente proporcional ao aumento da temperatura, na faixa de 18 a $32^{\circ} \mathrm{C}$. Geden (1996 e 1997) verificou que $S$. gemina foi mais sensível a extremos de temperatura do que Spalangia cameroni Perkins e $M$. raptor. Este último parasitóide, destruiu mais pupas de mosca doméstica, produziu mais descendentes e desenvolveu-se mais rapidamente que as outras espécies.

No Brasil existem muitos aviários, cujas aves produzem excrementos em quantidade, com grande proliferação de moscas, o que torna favorável o emprego de liberações de parasitóides para controle desses dípteros nocivos (Berti Filho et al., 1996). Informações básicas das principais espécies desses parasitóides ainda são escassas, à exceção de alguns trabalhos de levantamento populacional como os de Berti Filho et al. (1989), Pinheiro et al. (1989), Bruno et al. (1992), Sereno \& Neves (1993), Monteiro (1995) e de biologia como o de Costa (1995). O parasitóide Muscidifurax uniraptor, espécie partenogenética telítoca descrita por Kogan \& Legner (1970) ocorre em aviários do Estado de São Paulo (Berti Filho et al., 1989) parasitando pupas de mosca doméstica. Não há informações a respeito do potencial de parasitismo desse inimigo natural e de suas características biológicas em diferentes condições de temperatura, visando subsidiar uma possível utilização do mesmo para criação em laboratório e liberação em aviários.

O objetivo deste trabalho foi determinar a duração do ciclo biológico, as exigências térmicas e o parasitismo de $M$. uniraptor em pupas de Musca doméstica L., sob diferentes regimes de temperaturas constantes.

\section{MATERIAL E MÉTODOS}

No Departamento de Entomologia, Fitopatologia e Zoologia Agrícola da Escola Superior de Agricultura "Luiz de Queiroz" - Universidade de São Paulo (ESALQ/ USP), foi verificado o efeito da temperatura sobre 0 desenvolvimento do parasitóide $M$. uniraptor em câmaras climatizadas com $70 \pm 10 \%$ U.R. e $14 \mathrm{~h}$ de fotofase. Estas câmaras foram reguladas a 18, 20, 22, 25, 28, 30 e $32^{\circ} \mathrm{C}$, com variação de $\pm 1^{\circ} \mathrm{C}$.

A criação do hospedeiro, $M$. domestica, foi adaptada da metodologia de Costa (1995). Os adultos foram mantidos em gaiolas teladas de $70 \times 50 \times 40 \mathrm{~cm}$, alimentados com uma mistura seca de leite em pó e açúcar, fornecendo-se também água. Os ovos de $M$. domestica eram depositados em saquinhos de pano preto umedecidos com solução de leite em pó, levedura seca de cerveja e bicarbonato de amônio. A dieta das larvas consistiu de ração para coelhos, farelo de trigo e água. As condições ambientais da criação foram de 28 $\pm 1^{\circ} \mathrm{C}, 70 \pm 10 \%$ U.R. e $14 \mathrm{~h}$ de fotofase. O peso das pupas produzidas para utilização nos experimentos variou de 16 a $21 \mathrm{mg}$, sendo padronizado através de peneira de $2 \mathrm{~mm}$.

O parasitóide foi coletado na granja Mizumoto, região de Sorocaba, SP, através da exposição, a campo, de pupas de mosca doméstica produzidas em laboratório, conhecidas como pupas sentinelas (Rutz, 1986). As pupas parasitadas foram trazidas para 0 laboratório e os indivíduos de $M$. uniraptor que emergiram foram colocados em gaiolas teladas de $50 \mathrm{x}$ $50 \times 40 \mathrm{~cm}$, em sala climatizada $\left(25 \pm 1^{\circ} \mathrm{C}, 70 \pm 10 \%\right.$ U.R. e $14 \mathrm{~h}$ de fotofase), tendo como alimento pupas de mosca doméstica, trocadas a cada dois dias.

No laboratório, 200 pupas de mosca doméstica, com 24 a $48 \mathrm{~h}$ de idade, foram acondicionadas em tubos de vidro de $8,5 \mathrm{~cm}$ de comprimento $\times 2,5 \mathrm{~cm}$ de diâmetro, que foram tampados com algodão e levados para as respectivas câmaras. Parasitóides, com 0 a 24 h de idade, também foram agrupados em tubos de vidro com as mesmas dimensões e levados para essas câmaras, em grupos de 40 indivíduos por temperatura.

Após um período de aproximadamente uma hora, necessário para que a temperatura dos tubos se estabilizasse com a do interior da câmara, foi iniciado o processo de parasitismo. O hospedeiro foi exposto ao parasitóide, em cada temperatura, numa relação parasitóide: hospedeiro de 1:5, determinada em testes preliminares (seguindo a mesma metodologia) como mais eficiente (eficiência medida em termos de porcentagem de parasitismo e emergência de descendentes). Após $24 \mathrm{~h}$ em contato com as pupas, os parasitóides foram retirados e o material foi observado diariamente, descartando-se as moscas que emergiram e anotando-se a duração do período de ovo a adulto, o intervalo de emergência e o número de parasitóides emergidos nas diferentes temperaturas, considerando-se que de cada pupa emerge um parasitóide (espécie solitária). Foi verificada também a capacidade de parasitismo de $M$. uniraptor nas diferentes temperaturas. A mortalidade natural do hospedeiro foi observada para corrigir o parasitismo (Abbott, 1925), submetendo-se pupas de M. domestica às mesmas condições ambientais dos tratamentos.

Realizou-se análise de variância (ANOVA) com os valores da duração do período de ovo a adulto (o valor, em dias, da duração do ciclo de cada parasitóide emergido foi usado como repetição em cada tratamento, assim, cada temperatura [tratamento] teve número diferente de repetições). As médias obtidas em cada temperatura foram comparadas pelo teste de Tukey $(P \leq 0,05)$. Foram determinadas a temperatura base (limite térmico inferior de desenvolvimento) e a constante térmica para o período de ovo a adulto de fêmeas de M. uniraptor, através do método da hipérbole (Haddad \& Parra, 1984), utilizando o programa computacional MOBAE (Modelos Bioestatísticos Aplicados à Entomologia) (Haddad et al., 1995). 


\section{RESULTADOS E DISCUSSÃO}

A duração média do período de ovo a adulto de M. uniraptor decresceu com o aumento da temperatura, no intervalo de 18 a $30^{\circ} \mathrm{C}$, mas, a $32^{\circ} \mathrm{C}$ o efeito foi contrário, com aumento na duração do ciclo (TABELA 1). Geden (1997) também verificou que o tempo de desenvolvimento aumentou quando a temperatura passou de 30 para $32,5^{\circ} \mathrm{C}$, para S. gemina e S. cameroni e de 32,5 para $35^{\circ} \mathrm{C}$, para $M$. raptor. Provavelmente, essa mudança na tendência de diminuição do ciclo esteja relacionada ao afastamento da zona ótima de desenvolvimento para a espécie, interferindo na sua biologia.

O parasitóide $M$. raptor completou seu ciclo, a $18^{\circ} \mathrm{C}$, em 43 dias (Ables et al., 1976), cinco dias a menos que o observado para $M$. uniraptor (TABELA 1). Já, para $S$. endius e fêmeas de $S$. gemina, nessa mesma temperatura, o período de ovo a adulto foi de 66 (Ables et al., 1976) e 70,4 dias (Costa, 1995), respectivamente. $\mathrm{Na}$ temperatura de $15^{\circ} \mathrm{C}$, não houve emergência de $S$. gemina ou $S$. cameroni; enquanto $M$. raptor foi menos afetado (23,6\% de mortalidade) em relação aos outros parasitóides (100\% de mortalidade), pela exposição contínua a essa temperatura (Geden, 1997). Esses resultados demonstram as diferenças na duração do ciclo biológico entre as espécies de parasitóides de dípteros sinantrópicos, até dentro do mesmo gênero.

A tendência para $M$. raptor, $S$. endius e $S$. gemina foi de diminuir o ciclo nas temperaturas mais elevadas. Desse modo, a $32^{\circ} \mathrm{C}, M$. raptor levou apenas 15 dias para completar o ciclo (Ables et al., 1976), contra 18,3 dias para M. uniraptor (TABELA 1). Para S. endius esse valor ficou em 18 dias (Ables et al., 1976) e em 20,4 dias para fêmeas de S. gemina (Costa, 1995). Geden (1997) verificou que fêmeas de $S$. gemina, $S$. cameroni e $M$. raptor completaram o ciclo, a $32,5^{\circ} \mathrm{C}$, em $21,1,19,4$ e 13,8 dias, respectivamente. Contudo, segundo o autor, a exposição contínua a $35^{\circ} \mathrm{C}$ foi letal para os imaturos de $S$. gemina e matou a maioria de $S$. cameroni e $M$. raptor.

O período de ovo a adulto, a $25^{\circ} \mathrm{C}$, para $M$. uniraptor foi, em média, de 21,7 dias (TABELA 1). Este valor, nesta mesma temperatura, para fêmeas de outros parasitóides que se desenvolvem em pupas de mosca doméstica foi, em média, de 20 (Morgan et al., 1979) e 19,5 dias (Geden, 1997) para M. raptor, 22 (Morgan et al., 1991), 25,4 (Costa, 1995) e 27,8 dias (Geden, 1997) para S. gemina e 30 (Morgan et al., 1989) e 27,1 dias para S. cameroni (Geden, 1997).

Em todas as temperaturas a emergência dos descendentes ocorreu dentro de um período de tempo chamado de intervalo de emergência (TABELA 1). Este intervalo, em geral, assume a forma aproximada de uma hipérbole com uma abcissa várias vezes mais longa que o período de oviposição, quando este é limitado a $24 \mathrm{~h}$ (Legner, 1969). Costa (1995) verificou que à medida em que a temperatura aumentava o intervalo de emergência de $S$. gemina diminuia, o que não ocorreu com $M$. uniraptor (TABELA 1). Hipóteses para explicar a existência desse intervalo de emergência podem ser inferidas daquelas relatadas por Legner (1969), variando desde um desenvolvimento diferenciado das fases imaturas, superparasitismo, tendência diferenciada dos adultos formados em sair do hospedeiro, ou até a combinação destas. $O$ intervalo de emergência para $M$. uniraptor, a $25^{\circ} \mathrm{C}$, foi de 19 a 27 dias (TABELA 1), valor superior ao relatado por Kogan \& Legner (1970), 17 a 22 dias, cujos estudos foram realizados a $26 \pm 2^{\circ} \mathrm{C}$, umidade em $50 \%$ e fotofase de $13 \mathrm{~h}$.

De acordo com o teste $\chi^{2}\left(\chi^{2}=1,2839\right)$, que foi não significativo $(P \leq 0,05)$, os valores da duração do ciclo não diferiram daqueles estimados pela equação $(1 / D=$ $0,0257+0,0027 X$, onde $1 / D=$ velocidade de desenvolvimento e $X=$ temperatura em ${ }^{\circ} \mathrm{C}$ ), com coeficiente de determinação de 0,9306. Foi encontrado para temperatura base (limiar térmico inferior de desenvolvimento) o valor de $9,43^{\circ} \mathrm{C}$, com constante térmica de 366,62 GD, onde GD= graus-dia. Com os valores estimados construiu-se também um gráfico da duração do ciclo e da velocidade de desenvolvimento em função da temperatura (Figura 1).

Os valores de porcentagem de parasitismo e de emergência de descendentes foram aumentando com a temperatura, até atingirem 0 máximo a $28^{\circ} \mathrm{C}$, decrescendo a temperaturas maiores, principalmente a porcentagem de emergência de descendentes. Essa diminuição na emergência da progênie pode ter ocorrido devido a um maior ressecamento das pupas nas temperaturas mais altas (Figura 2). O número de parasitóides emergidos também foi afetado da mesma maneira (TABELA 1). Geden (1996) verificou que o desempenho reprodutivo de $S$. gemina, $S$. cameroni e M. raptor aumentou com a elevação da temperatura, dentro da faixa de 15 a $30^{\circ} \mathrm{C}$, decrescendo a $35^{\circ} \mathrm{C}$ Essas espécies de parasitóides destruíram mais pupas e produziram mais descendentes a $30^{\circ} \mathrm{C}$ do que a 15,20 , 25 ou $35^{\circ} \mathrm{C}$. M. raptor produziu mais descendentes que os outros parasitóides.

Considerando-se os dois extremos de temperatura testados, a $18^{\circ} \mathrm{C}$ a capacidade reprodutiva de $M$. uniraptor foi mais afetada do que a $32^{\circ} \mathrm{C}$ (Figura 2). No entanto, outras espécies de parasitóides não se reproduzem nessas temperaturas mais baixas. Segundo Ables \& Shepard (1976) S. endius não ovipositou a 12,8 e $18^{\circ} \mathrm{C}$, por causa da redução na atividade locomotora das fêmeas. Contudo, $M$. raptor ovipositou nessas temperaturas, embora com redução no parasitismo. Esses resultados estão de acordo com o que foi encontrado por Legner (1977), que verificou que as espécies de Muscidifurax, no caso $M$. uniraptor, em temperaturas mais baixas, têm desempenho reprodutivo superior a muitas espécies de parasitóides de dípteros sinantrópicos. 
TABELA 1 - Duração (média \pm erro padrão) do período de ovo a adulto e intervalo de emergência de fêmeas de $M$. uniraptor e número de parasitóides emergidos em pupas de $M$. domestica, em sete temperaturas ( $70 \pm 10 \%$ U.R. e $14 \mathrm{~h}$ de fotofase).

\begin{tabular}{|c|c|c|c|}
\hline Temperatura & Duração & $\begin{array}{l}\text { Intervalo de } \\
\text { emergência }\end{array}$ & $\begin{array}{l}\text { Número de } \\
\text { parasitóides } \\
\text { emergidos }\end{array}$ \\
\hline${ }^{\circ} \mathrm{C}$ & \multicolumn{3}{|c|}{ 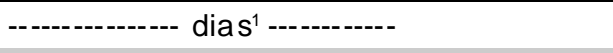 } \\
\hline 18 & $48,0 \pm 0,37 a$ & $46-53$ & 22 \\
\hline 20 & $36,5 \pm 0,12 b$ & $35-39$ & 69 \\
\hline 22 & $28,7 \pm 0,17 c$ & $27-35$ & 72 \\
\hline 25 & $21,7 \pm 0,10 d$ & $19-27$ & 116 \\
\hline 28 & $18,3 \pm 0,10 \mathrm{e}$ & $17-24$ & 127 \\
\hline 30 & $17,0 \pm 0,09 f$ & $15-21$ & 120 \\
\hline 32 & $18,3 \pm 0,13 \mathrm{e}$ & $16-22$ & 89 \\
\hline
\end{tabular}

${ }^{1}$ Médias seguidas pela mesma letra não diferem pelo teste de Tukey $(P \leq 0,05)$.

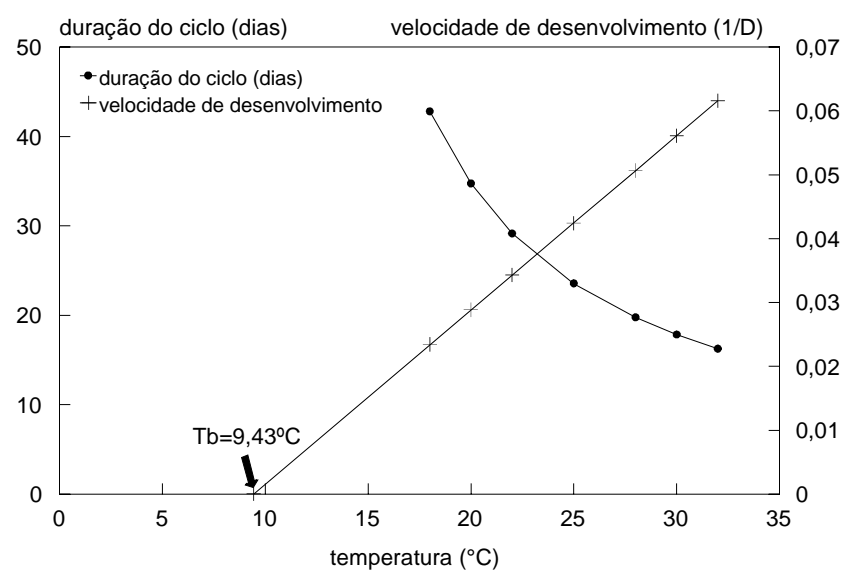

Figura 1 - Duração do ciclo (ovo a adulto) e velocidade de desenvolvimento de $M$. uniraptor, em pupas de $M$. domestica, em função da temperatura $(70 \pm 10 \%$ U.R. e $14 \mathrm{~h}$ de fotofase). $\mathrm{Tb}=$ temperatura base.

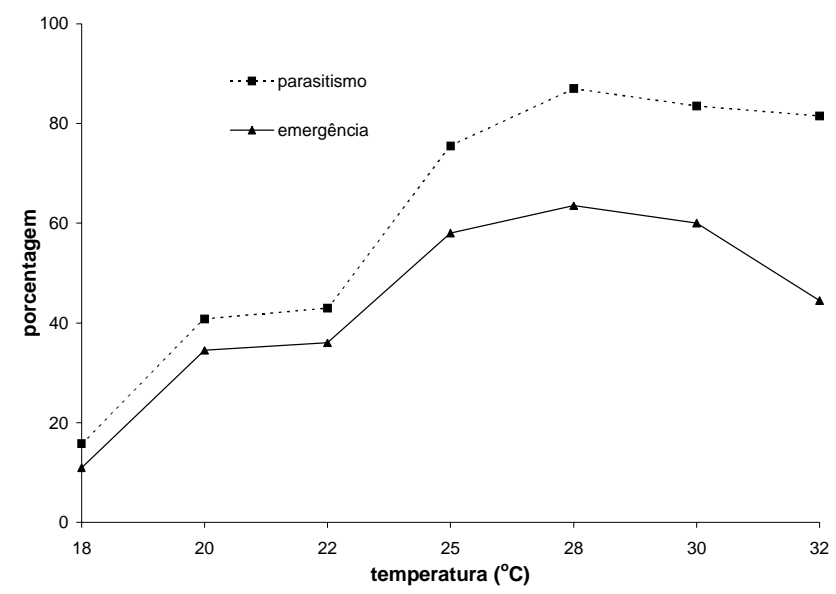

Figura 2 - Porcentagem de parasitismo e de emergência de descendentes de $M$. uniraptor em pupas de $M$. domestica em sete temperaturas constantes $(70 \pm 10 \%$ U.R. e $14 \mathrm{~h}$ de fotofase)
Analisando-se conjuntamente os valores de duração do ciclo, número de descendentes e capacidade reprodutiva, fatores estes, dentre outros, que medem a eficiência de um parasitóide, a faixa de temperatura entre 28 e $30^{\circ} \mathrm{C}$ mostrou-se a mais adequada para criação do parasitóide $M$. uniraptor em pupas de mosca doméstica.

\section{REFERÊNCIAS BIBLIOGRÁFICAS}

ABBOTT, W.S. A method of computing the effectiveness of an insecticide. Journal of Economic Entomology, v.18, p.265-267, 1925.

ABLES, J.R.; SHEPARD, M. Influence of temperature on oviposition by the parasites Spalangia endius and Muscidifurax raptor. Environmental Entomology, v.5, p.511-513, 1976.

ABLES, J.R.; SHEPARD, M.; HOLMAN, J.R. Development of the parasitoids Spalangia endius and Muscidifurax raptor in relation to constant and variable temperature: simulation and validation. Environmental Entomology, v.5, p.329-332, 1976.

AXTELL, R.C. Fly control in confined livestock and poultry production. Greensboro: Ciba-Geigy, 1986. 59p.

AXTELL, R.C.; ARENDS, J.J. Ecology and management of arthropod pests of poultry. Annual Review of Entomology, v.35, p.101-126, 1990.

BERTI FILHO, E.; COSTA, V.A.; AAGESEN, T.L. Occurrence of natural enemies of Musca domestica L. (Diptera: Muscidae) in poultry areas of Bastos, State of São Paulo, Brazil. Revista de Agricultura, v.64, p.98, 1989.

BERTI FILHO, E.; THOMAZINI, M.J.; COSTA, V.A. Artrópodes benéficos associados ao esterco de galinhas poedeiras no Brasil. Revista de Agricultura, v.71, p.273-286, 1996.

BRUNO, T.V.; GUIMARÃES, J.H.; TUCCI, E.C.; SANTOS, A.M.M. dos. Parasitóides associados a dípteros sinantrópicos de granjas de aves de postura no Estado de São Paulo, Brasil. Revista Brasileira de Parasitologia Veterinária, v.1, p.55-59, 1992.

COSTA, V.A. Efeito da temperatura na biologia de Spalangia gemina Boucek, 1963 (Hymenoptera, Pteromalidae), parasitóide pupal de Musca domestica L., 1758 (Diptera, Muscidae). Piracicaba, 1995. 67p. Tese (Doutorado) - Escola Superior de Agricultura "Luiz de Queiroz", Universidade de São Paulo.

GEDEN, C.J. Modeling host attacks and progeny production of Spalangia gemina, Spalangia cameroni, and Muscidifurax raptor (Hymenoptera: Pteromalidae) at constant and variable temperatures. Biological Control, v.7, p.172-178, 1996.

GEDEN, C.J. Development models for the filth fly parasitoids Spalangia gemina, S. cameroni, and Muscidifurax raptor (Hymenoptera: Pteromalidae) under constant and variable temperatures. Biological Control, v.9, p.185-192, 1997.

HADDAD, M.L.; PARRA, J.R.P. Métodos para estimar os limites térmicos e a faixa ótima de desenvolvimento das diferentes fases do ciclo evolutivo dos insetos. Piracicaba: FEALQ, 1984. 12p.

HADDAD, M.L.; MORAES, R.C.B.; PARRA, J.R.P. Sistema computadorizado para modelos bioestatísticos aplicados à entomologia. In: CONGRESSO BRASILEIRO DE ENTOMOLOGIA, 15., Caxambu, 1995. Resumos. Caxambu: Seb/Esal, 1995. p.244.

KOGAN, M.; LEGNER, E.F. A biosystematic revision of the genus Muscidifurax (Hymenoptera: Pteromalidae) with descriptions of four new species. Canadian Entomologist, v.102, p.1268-1290, 1970.

LEGNER, E.F. Adult emergence interval and reproduction in parasitic Hymenoptera influenced by host size and density. Annals of the Entomological Society of America, v.62, p.220-226, 1969. 
LEGNER, E.F. Temperature, humidity, and depth of habitat influencing host destruction and fecundity of muscoid fly parasites. Entomophaga, v.22, p.199-206, 1977.

MONTEIRO, M.R. Microhimenópteros (Insecta: Hymenoptera) parasitóides e insetos predadores de mosca sinantrópicas (Insecta: Diptera) na Granja Capuavinha, Monte Mor, SP. Campinas, 1995. 98p. Dissertação (Mestrado) - Universidade Estadual de Campinas.

MORGAN, P.B.; WEIDHASS, D.E.; LABRECQUE, G.C. Host-parasite relationship of the house fly, Musca domestica L., and the microhymenopteran pupal parasite, Muscidifurax raptor Girault and Sanders (Diptera: Muscidae and Hymenoptera: Pteromalidae). Journal of the Kansas Entomological Society, v.52, p.276-281, 1979.

MORGAN, P.B.; HOYER, H.; PATTERSON, R.S. Life history of Spalangia cameroni (Hymenoptera: Pteromalidae), a microhymenopteran pupal parasite of muscoid flies (Diptera: Muscidae). Journal of the Kansas Entomological Society, v.62, p.381-386, 1989.
MORGAN, P.B.; BERTI FILHO, E.; COSTA, V.A. Life history of Spalangia gemina Boucek (Hymenoptera: Pteromalidae), a fast-breeding microhymenopteran pupal parasitoid of muscoid flies. Medical Veterinary Entomology, v.5, p.277-281, 1991.

PINHEIRO, J.B.; BUENO, V.H.P.; ALVES, D.G. Ocorrência de parasitóides de Musca domestica L., 1758 (Diptera: Muscidae) em Lavras, Minas Gerais. Anais da Sociedade Entomológica do Brasil, v.18, p.419-421, 1989.

RUTZ, D.A. Parasitoid monitoring and impact evaluation in the development of filth fly biological control programs for poultry farms. In: PATTERSON, R.S.; RUTZ, D.A. (Ed.) Biological control of muscoid flies. College Park: Entomological Society of America, 1986. p.45-51. (Miscellaneous Publications, 61).

SERENO, F.T.P.S.; NEVES, D.P. Ocorrência natural de microhimenópteros parasitóides de pupas de moscas em aviários. Anais da Sociedade Entomológica do Brasil, v.22, p.527-533, 1993.

Recebido em 11.05 .00 